\title{
Panorama mundial das pesquisas com robôs subaquáticos remotamente operados (ROV)
}

\author{
World panorama of remotely operated underwater robots (ROV) research \\ Panorama mundial de la investigación de robots submarinos operados a distancia (ROV)
}

Recebido: 10/09/2021 | Revisado: 16/09/2021 | Aceito: 22/09/2021 | Publicado: 25/09/2021

\author{
Amanda Aparecida Carminatto \\ ORCID: https://orcid.org/0000-0002-3765-7992 \\ Universidade Federal de São Carlos, Brasil \\ Núcleo de Tecnologia Marinha e Ambiental, Brasil \\ E-mail: amandacarminatto@gmail.com \\ Giovana Ciongoli \\ ORCID: https://orcid.org/0000-0002-8007-3394 \\ Instituto de Pesquisas Energéticas e Nucleares, Brasil \\ Núcleo de Tecnologia Marinha e Ambiental, Brasil \\ E-mail: giciongoli@usp.br \\ Gaianê Sabundjian \\ ORCID: https://orcid.org/0000-0001-9544-4509 \\ Instituto de Pesquisas Energéticas e Nucleares, Brasil \\ E-mail: gdjian@ipen.br
}

\begin{abstract}
Resumo
Veículos subaquáticos operados remotamente (ROVs) fazem parte de um grupo de tecnologias que vem sendo cada vez mais utilizados como ferramenta em pesquisas científicas. As aplicações para esses veículos são vastas e o trabalho tem por objetivo apresentar análises de âmbito socioeconômico e ambiental trazendo um panorama mundial sobre as pesquisas científicas que incluem os ROVs. Foi criada uma matriz de dados com pesquisa literária sendo incluídos 78 documentos publicados. As principais informações avaliadas foram continentes e países que publicam trabalhos, parcerias realizadas entre os países, se houve ou não financiamento para a realização do projeto, qual a área de estudo e aplicação e quais as características do ROV utilizado, especialmente se ele foi desenvolvido exclusivamente para o projeto ou se houve aquisição de equipamento já existente. Notamos que os países mais desenvolvidos são os que mais investem na tecnologia, tanto em número de publicações quanto em financiamentos, fato esse que pode ser justificado pelo compromisso de as entidades governamentais fazerem aportes constantes na pesquisa científica. Em contrapartida, países em desenvolvimento como Brasil e China vem apresentando ótimos resultados e crescimento na aplicação de recursos em ciência e tecnologia. Os benefícios do investimento nesta tecnologia refletem em toda a sociedade, gerando empregos, contribuindo para a economia nacional e incentivando a pesquisa, o conhecimento e a ciência.
\end{abstract}

Palavras-chave: Tecnologia; Investimento; Socioeconômico; Ambiental; Energia nuclear.

\begin{abstract}
Remotely operated underwater vehicles (ROVs) are part of a group of technologies that have been increasingly used as a tool in scientific research. The applications for these vehicles are vast and the work aims to present analyzes of socioeconomic and environmental scope, bringing a world panorama on scientific research that includes ROVs. A data matrix with literary research was created, including 78 published documents. The main information evaluated were continents and countries that publish works, partnerships between countries, whether there was funding for the project, the area of study and application and the characteristics of the ROV used, especially if it was developed exclusively for the project or if existing equipment was purchased. We note that the most developed countries are the ones that invest the most in technology, both in number of publications and in funding, a fact that can be justified by the commitment of government entities to make constant contributions to scientific research. On the other hand, countries like Brazil and China have been showing excellent results and growth in the application of resources in science and technology. The benefits of investing in this technology are reflected throughout society, generating jobs, contributing to the national economy, and encouraging research, knowledge, and science.
\end{abstract}

Keywords: Technology; Investment; Socioeconomic; Environmental; Nuclear energy.

\section{Resumen}

Los vehículos submarinos operados a distancia (ROV) forman parte de un grupo de tecnologías que se han utilizado cada vez más como herramienta en la investigación científica. Las aplicaciones para estos vehículos son amplias y el trabajo tiene como objetivo presentar análisis de alcance socioeconómico y ambiental, trayendo un panorama mundial 
de la investigación científica que incluye los ROV. Se elaboró una matriz de datos con investigación literaria, que incluye 78 documentos publicados. La principal información evaluada fueron continentes y países que publican trabajos, alianzas entre países, si hubo o no financiamiento para el proyecto, cuál es el área de estudio y aplicación y cuáles son las características del ROV utilizado, especialmente si fue desarrollado exclusivamente para el proyecto o si se compró el equipo existente. Observamos que los países más desarrollados son los que más invierten en tecnología, tanto en número de publicaciones como en financiamiento, hecho que puede justificarse por el compromiso de las entidades gubernamentales de realizar contribuciones constantes a la investigación científica. Por otro lado, países en desarrollo como Brasil y China vienen mostrando excelentes resultados y crecimiento en la aplicación de recursos en ciencia y tecnología. Los beneficios de invertir en esta tecnología se reflejan en toda la sociedad, generando puestos de trabajo, contribuyendo a la economía nacional y fomentando la investigación, el conocimiento y la ciencia.

Palabras clave: Tecnología; Inversión; Socioeconómico; Ambiental; Energía nuclear.

\section{Introdução}

Veículos subaquáticos comumente se dividem em duas categorias: veículos tripulados e veículos não tripulados. Inseridos na última categoria estão os AUVs (Veículos Subaquáticos Autônomos) e os ROVs (Veículos Subaquáticos Controlados Remotamente). O que o difere por sua inteligência artificial, presente nos veículos autônomos, e pela presença de um cabo umbilical responsável pela comunicação constante entre o piloto e o veículo, no caso dos ROVs (Christ \& Wernli, 2014).

Na literatura geral, a nomenclatura "Veículos Operados Remotamente" pode ser aplicada a todo e qualquer sistema robótico controlado por um operador à distância, como por exemplos os veículos aéreos não tripulados (Cooke, 2006). Contudo, na literatura específica, o termo ROV, sigla em inglês para Remotely Operated Vehicle, refere-se exclusivamente a sistemas subaquáticos (Christ \& Wernli, 2014, Tahir \& Iqbal, 2014).

De maneira simplista e considerando uma configuração básica para um ROV ser denominado e classificado assim, um veículo subaquático tripulado é um compartimento estanque que contém câmeras de vídeo, luzes e propulsores. Todos esses componentes são manipulados por um operador que, em tempo real, envia sinais de controle através de um cabo chamado de umbilical e recebe os dados de vídeo e informações em um monitor. A comunicação via telemetria requer que um operador execute as tarefas a todo momento, de maneira intuitiva e técnica (Christ \& Wernli, 2014, NUTECMAR, 2020).

Assim como a robótica geral, o desenvolvimento das tecnologias subaquáticas são constantes e crescem conforme aumentam as demandas e necessidades. Os ROVs vão de simples sistemas básicos e compactos à sistemas complexos que atingem a maior profundidade dos oceanos e, portanto, o grau requerido de sofisticação e conhecimento de seus operadores aumenta à medida que a tecnologia se desenvolve e torna-se cada vez mais reconhecida e aplicável (Christ \& Wernli, 2014, NUTECMAR, 2020).

Christ e Wernli (2014) utilizaram o tamanho dos veículos e suas capacidades como principais características e publicou a seguinte classificação para os ROVs:

- Observation Class Remolely Operated Vehicle (OCROV) ou Classe de Observação: comumente são de pequeno porte e permitem que seu uso seja feito por uma equipe reduzida, não sendo necessário embarcações de grande porte ou estruturas mais complexas em sua operação.

- Mid-sized Remotely Operated Vehicle (MSROV) ou Veículos de Médio Porte ou Compactos: é a categoria mais abrangente em termos de tamanho e peso de veículos pois apresenta os ROVs com estruturas mecânicas mais robustas e, portanto, preparadas para suportar maiores pressões e maiores pesos.

- Work Class Remotely Operated Vehicles (WCROV) ou Classe de Trabalho: são mecanicamente maiores, seus componentes são mais potentes e precisos, seus manipuladores são hidráulicos e não elétricos como nas demais categorias e se faz necessário equipe especializada na operação que ocorre preferencialmente a partir de uma plataforma ou embarcação de grande porte. 
- Special-use vehicles ou Veículos Especiais: os veículos dessa categoria são rebocados ou possuem lagartas para se locomover sobre o solo marinho.

A aplicabilidade dos ROVs é ampla e abrange os mais diversos mercados mundiais e a escolha do equipamento ideal está diretamente relacionada à eficiência de sua utilização nos mais variados ambientes à que podem ser expostos (Tahir \& Iqbal, 2014, NUTECMAR, 2020).

De exploração dos oceanos a investigações de estruturas subaquáticas, passando por estudos geológicos e operações militares, os ROVs exercem um importante papel dentro de cada possibilidade apresentada e são ferramentas cada vez mais aplicadas e valorizadas pela indústria comercial, governamental, na área nuclear, militar e acadêmica (Tahir \& Iqbal, 2014, Zarei et al., 2020).

O intuito do presente estudo é apresentar um panorama mundial sobre as pesquisas científicas que envolvem a ferramenta tecnológica ROV, por meio da i) perspectiva histórica dos estudos com ROVs e suas aplicações, ii) estudos realizados por meio de parcerias e financiamento e iii) classificação e nacionalidade dos ROVs.

\section{Metodologia}

\subsection{Revisão integrativa de literatura}

Foi utilizada a revisão integrativa de literatura, que permite sumarizar pesquisas anteriores e delas obter conclusões gerais para analisar o conhecimento científico sobre o assunto a ser investigado (Souza et al., 2010). O levantamento bibliográfico foi realizado nas bibliotecas eletrônicas SciELO, Web of Science, Scopus e na base de dados do Google Acadêmico. Para tal, considerou-se os trabalhos que utilizam o ROV e/ou acessórios para ROV como instrumento de pesquisa in situ e ex situ em atividades subaquáticas. Os trabalhos indexados duplicados e aqueles que não tratavam o ROV como assunto principal foram desconsiderados.

\subsection{Coleta de informações}

Cada documento foi inserido em um banco de dados com autor (es), ano de publicação e os seguintes campos:

- Continente e país: referente a nacionalidade dos autores/pesquisadores;

- Parceria: quando há mais de uma afiliação no trabalho;

- Financiamento: se houve algum e qual o tipo de órgão de fomento: governamental ou privado;

- Área de estudo: engenharia ou ciência ambiental;

- Aplicação: estudo de engenharia, energia nuclear, organismos marinhos, petróleo e gás, estruturas submersas, geologia marinha e poluição marinha;

- ROV: se o objeto de trabalho é o desenvolvimento de um ROV ou se o ROV utilizado já existe comercialmente;

- Nacionalidade do ROV: referente ao fabricante dos equipamentos.

- Classe de ROV: OCROV (robô para observação), MSROV (robô para pequenas intervenções) e WCROV (robô para trabalho) de acordo com Christ \& Wernli (2014).

- Tipo de documento: se o trabalho foi publicado em formato de artigo científico, congressos/conferências/encontro, trabalho de conclusão de curso, dissertação ou tese.

Os mapas que contribuíram com a análise dos dados foram realizados no programa Arcgis 10.6. 


\section{Resultados}

\subsection{Perspectiva histórica dos estudos com ROVs e suas aplicações}

A compilação dos trabalhos sobre ROV totalizou 78 documentos publicados entre 1998 até o primeiro semestre de 2020. A Figura 1 indica um aumento exponencial no número de publicações nos últimos 25 anos.

Figura 1. Número de trabalhos anuais sobre ROV publicados entre 1998 e 2020.

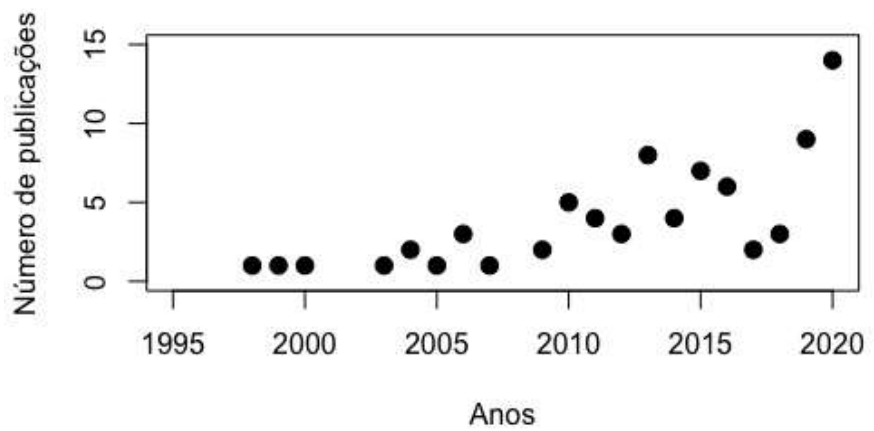

Fonte: Autores.

Considerando os tipos de publicações, 78\% ( $\mathrm{n}=61$ ) foram em forma de artigo científico, 14,1\% (n=11) apresentados em conferências/congressos/encontros científicos e 6,7\% (n=5) trabalhos resultantes de graduação e mestrado.

$\mathrm{O}$ avanço em trabalhos utilizando os ROVs envolveu as áreas de estudo da engenharia/tecnologia e ciências ambientais e nas mais diversas aplicações. A área de ciências ambientais foi responsável pelo maior número de trabalhos publicados $(59 \%, \mathrm{n}=46)$. Nas aplicações, organismos marinhos $(43,5 \%, \mathrm{n}=34)$ e engenharia $(27 \%, \mathrm{n}=21)$ englobaram a maioria dos trabalhos, seguidos por geologia marinha $(11,5 \%, n=9)$, engenharia nuclear $(9 \%, n=7)$, estruturas submersas $(3,9 \%, n=3)$, poluição marinha $(3,9 \%, \mathrm{n}=3)$ e petróleo e gás $(1,2 \%, \mathrm{n}=1)$ (Tabela 1$)$.

Tabela 1. Número de estudos utilizando o ROV categorizados por área e aplicação.

\begin{tabular}{lll}
\hline Área de estudo & Aplicação & Total \\
\hline \multirow{3}{*}{ Ciência ambiental } & Organismos marinhos & 34 \\
& Geologia marinha & 9 \\
& Poluição marinha & 3 \\
\hline \multirow{4}{*}{ Engenharia e tecnologia } & Engenharia & 21 \\
& Energia nuclear & 7 \\
& Estruturas submersas & 3 \\
& Petróleo e Gás & 1 \\
\hline
\end{tabular}

Fonte: Autores.

Os EUA, Brasil, Reino Unido, França e Japão se destacaram por utilizem os ROVs em estudos em diversas aplicações e áreas como mostra a Figura 2.

Os EUA concentraram seus trabalhos utilizando o ROV na área da ciência ambiental $(82,3 \%, n=14)$, em pesquisas relacionadas a estudos com organismos marinhos $(57,1 \%, \mathrm{n}=8)$, geologia marinha $(35,7 \%, \mathrm{n}=5)$ e poluição marinha $(7,1 \%$, $\mathrm{n}=1)$. Também dedicou uma parte na área de engenharia/tecnologia $(17,7 \%, \mathrm{n}=3)$, aplicando em estudos relacionados ao desenvolvimento de novas tecnologias.

O Brasil investiu em trabalhos tanto na área de ciência ambiental $(53,9 \%, n=7)$ quanto em engenharia/tecnologia 
$(46,1 \%, n=6)$ e nas diversas aplicabilidades, principalmente em pesquisa com organismos marinhos $(85,7 \%$, $n=6)$ e na engenharia $(85,7 \%, \mathrm{n}=5)$ desenvolvendo novas tecnologias para os ROVs.

O Reino Unido concentrou seus estudos apenas na área de ciência ambiental, especificamente em estudos com organismos marinhos $(62,5 \%, \mathrm{n}=5)$ e geologia marinha $(37,5 \%, \mathrm{n}=3)$. A França e a Itália seguiram na mesma linha, porém, a França publicou a maioria dos seus trabalhos na área de ciências ambientais $(75 \%, \mathrm{n}=3)$, focando em pesquisas com organismos marinhos $(66,7 \%, n=2)$ e em geologia marinha $(33,3 \%, n=1)$.

A China, diferente dos outros países, dedicou seus trabalhos na área de engenharia/tecnologia e $80 \%$ ( $\mathrm{n}=3$ ) em estudos envolvendo novas tecnologias para os ROVs e seus acessórios para o mercado de energia nuclear.

Figura 2. Número de aplicações de ROVs por países que produzem pesquisas científicas com a tecnologia.

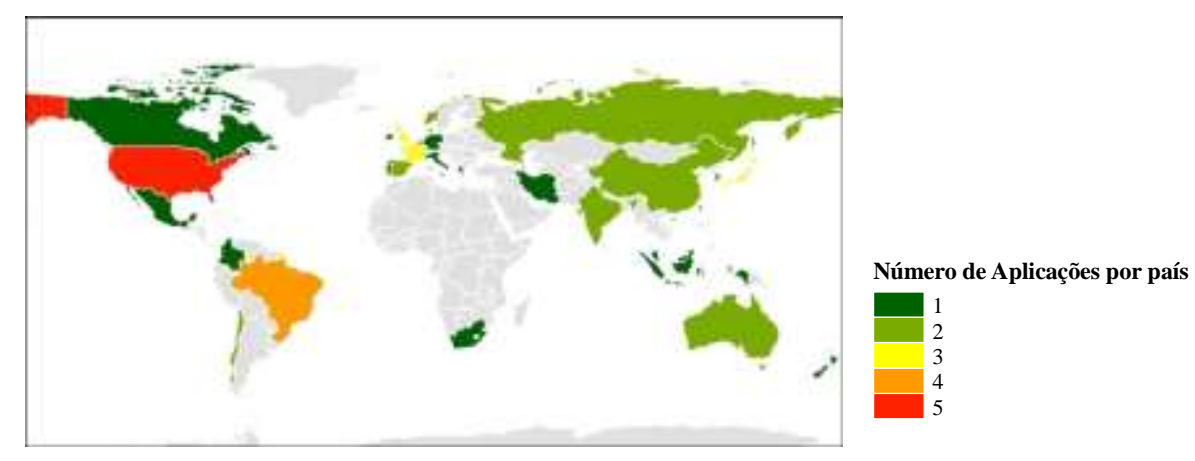

Fonte: Autores.

\subsection{Estudos com ROV, parcerias e financiamento}

Levando em consideração a nacionalidade dos autores e por continente, os trabalhos com ROVs nos países da América (norte, central e sul) foram os mais numerosos do mundo (40,6\% das publicações, n=41), seguindo pela Europa $(30,7 \%, n=31)$, Ásia $(22,8 \%, n=23)$, Oceania $(5 \%, n=5)$ e África $(1 \%, n=1)$.

Considerando o número de publicações por país, 31 países realizam estudos com o ROV, incluindo os EUA com 17 artigos (16,8\%), Brasil com 13 estudos (12,9\%), Reino Unido ( $\mathrm{n}=8 \%, \mathrm{n}=7,9)$, Itália (5\%, $\mathrm{n}=5)$, seguidos por China, Austrália, Chile, Colômbia, Coréia do Sul e França (4\%, n=4). Estes 10 países representam 66,3\% da publicação mundial.

Publicações que envolvem parceria entre vários países representam 24,4\% (n=19) realizadas principalmente pelos EUA $(12,5 \%, n=5)$, Reino Unido $(10 \%, n=4)$, Espanha $(7,5 \%, n=3)$ e Alemanha, Austrália, França, Índia, Japão, México e Rússia (5\%, n=2) (Figura 3).

Trabalhos que possuem financiamento representam 60,2\% (n=47). Destes, 76,6\% $(n=36)$ são financiados por órgão governamentais e $23,4 \%(n=11)$ por organizações privadas. Os países que mais se destacam por publicarem com algum tipo de financiamento são EUA $(16,7 \%, n=11)$, Brasil $(13,6 \%, n=9)$, Reino Unido $(9 \%, n=6)$, Chile $(6 \%$, $n=4)$, seguidos pela Alemanha, Colômbia, Espanha e Índia (4,5\%, n=3) (Figura 3). 
Figura 3. Mapa mundial com a interação entre os países que realizam pesquisas, suas parcerias e formas de financiamento.

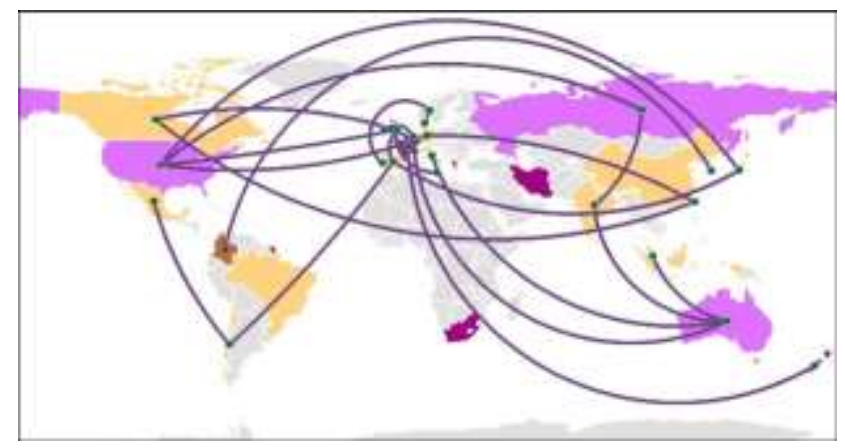

\section{Estudos, parcerias e fluanclamento de ROV}

- Paises com parceria

- Fluxo de parceria entre os paises

Sem financiamento

Financiamento governamental

Fiuanciamento privado

Financiatnento privado e governamental

Fonte: Autores.

\subsection{ROVs, classificação e nacionalidade}

Estudos que têm como objetivo o desenvolvimento de novos ROVs ou acessórios para o robô representam 33,3\% ( $\mathrm{n}=26)$. Em relação a nacionalidade destes equipamentos, o Brasil $(23 \%, \mathrm{n}=6)$, China $(15 \%, \mathrm{n}=4)$ e Colômbia se destacam $(12 \%, n=3)$ por desenvolverem novas tecnologias. Dentre eles, 87\% $(n=20)$ são classificados como robôs de observação (OCROV) e 13\% (n=3) como robôs de trabalho (WCROV). Todos esses 26 trabalhos estão dentro da área de engenharia/tecnologia e em três diferentes aplicações, se destacam engenharia $(73 \%, n=19)$ e energia nuclear $(23 \%$, $n=6)$ (Figura 4).

Figura 4. Utilização de ROVs desenvolvidos ou existentes por países que elaboram pesquisas científicas na área.

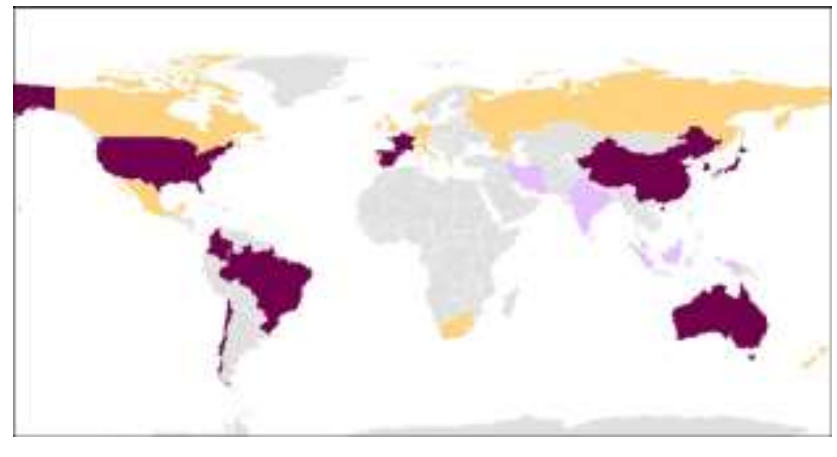

Utilização de ROVs desenvolvidos ou existentes

ROV existente no mercado

ROV desenvolvido em estudo

Misto

Fonte: Autores.

Estudos que utilizam ROVs já existente no mercado como ferramenta de pesquisa, representam 66,7\% (n=52). Os EUA $(44,9 \%, n=22)$, Reino Unido $(16,3 \%, n=8)$, França $(14,3 \%, n=7)$ e Itália $(6,1 \%, n=3)$ se destacam por serem grandes fabricantes mundiais de ROVs. Dentre eles, 44,2\% (n=23) são classificados como robôs de trabalho (WCROV), 30,8\% (n=16) como robôs de observação (OCROV) e 25\% (n=13) como robôs que executam pequenas intervenções (MSROV). Quase todos esses trabalhos estão dentro da área de ciência ambiental $(85,5 \%, \mathrm{n}=46)$ e em sete diferentes aplicações. As que mais se destacam são organismos marinhos $(65,4 \%, n=34)$, geologia marinha $(15,4 \%, n=9)$ e poluição marinha $(5,8 \%$, $n=3)($ Figura 4$)$.

\section{Discussão}

\subsection{Perspectiva histórica dos estudos com ROVs e suas aplicações}

Embora os ROVs tenham surgido a partir de 1950 (Tahir \& Iqbal, 2014), os dados mostram que os trabalhos científicos utilizando o ROV são relativamente novos, iniciando na década de 90. Preteritamente, essa tecnologia foi desenvolvida para fins militares e posteriormente usada na exploração de petróleo e gás. A marinha dos EUA é considerada 
uma das primeiras desenvolvedoras desta tecnologia. Na década de 1960 e 1970, eles desenvolveram os ROVs CURV, CURV II e CURV III para resgate e recuperação de munições (Lemaire, 1988, Tahir \& Iqbal, 2014). A partir da década de 1980, a indústria de petróleo e gás impulsionou a pesquisa e o desenvolvimento de tecnologias de ROV incentivando a inovação em uma variedade de campos (Tahir \& Iqbal, 2014). Hoje, ROVs são amplamente usados nas áreas de meio ambiente, engenharia, energia e são inúmeras as possibilidades de inserção de acessórios e ferramentas que aprimoram a aplicabilidade dos ROVs nas mais variadas demandas (Ho et al., 2011, NUTECMAR, 2020), desde trabalhos envolvendo a diversidade de organismos marinhos de forma não invasiva, estudo de formação e estrutura do fundo do mar, rastreamento, quantificação e qualificação de lixo marinho e trabalhos realizados em ambientes nucleares, como comprovam os nossos resultados apresentados neste trabalho.

Com o aumento das publicações a partir da década de 90, a ferramenta ROV passou a ser vista não somente em suas áreas de atuação, como também no meio acadêmico e científico. Por se tratar de uma tecnologia em crescente expansão e com aplicabilidade comercial, e não somente para fins científicos e militares, os trabalhos publicados são específicos, com nível de detalhamento e riqueza e em grande maioria, inéditos (Zhang et al., 2013, Lv et al., 2014, Luo et al., 2018, Boutteau et al. 2020, Htun et al., 2018, Wahab et al., 2020, Zhou \& Zhao, 2020).

Haja visto que $78 \%$ das publicações utilizadas são de artigos científicos, método de publicação com processos de aceitação mais rigorosos e técnicos do que se comparado aos demais tipos de publicações. Independentemente do teor de rigorosidade, publicações científicas, trabalhos apresentações em anais de congresso e dissertações sobre temas específicos como os ROVs tendem a aumentar, englobando cada vez mais áreas de pesquisa e aplicações diferentes (Centeno, 2007, Souza, 2010, Tehrani et al., 2010, Lapa et al., 2012, Alcoforado et al., 2013, Smolowitz et al., 2015, Carminatto, 2019).

Os EUA, Brasil, Reino Unido, França e a Itália são os países que mais investem em pesquisas científicas subaquáticas. Abrangem estudos relacionados à ambientes marinhos estuarinos e apresentam resultados que envolvem áreas tais como biologia, oceanografia, geologia e outras (Lorance \& Trenkel, 2006, Linley et al., 2013, Cánovas-Molinaet al., 2016, Carminatto et al., 2020, Hartill al., 2020, Johansen et al., 2020). Todos esses ambientes são constantemente afetados pelo aumento da influência antrópica, principalmente no ambiente marítimo e estuarino com a exploração dos recursos naturais (Hughes et al., 2010, Manoukian et al., 2011). Transformando as pesquisas científicas subaquáticas, os ROVs são ferramentas em constante inserção, pois trazem a possibilidade de as investigações ecológicas de ambientes aquáticos se tornarem experimentais e não invasivas, deixando de ser apenas observações históricas (Hughes et al., 2010).

Os países asiáticos, China, Coreia do Sul e Japão investem seus estudos desenvolvendo novas tecnologias subaquáticas, principalmente na área nuclear (Koji, 1999, Park et al., 2009, Zhang et al., 2013, Lv et al., 2014, Luo et al., 2018). O mercado industrial e principalmente as indústrias de energia que estão inseridas em um ambiente complexo e insalubre, admitiram o uso de veículos subaquáticos remotos em suas operações, à princípio porque contribuíam para o aumento da segurança e posteriormente por sua eficiência metodológica de inspeções e economia de recursos e tempo (Koji, 1999, Cho et al., 2004, Lee et al., 2006, Gao et al. 2011, Luo et al., 2018).

\subsection{Estudos com ROV, parcerias e financiamento}

Em nossos resultados, o continente americano é o mais representativo em publicações porque abrange os países da América do Norte, Sul e Central sendo os EUA e Brasil os países líderes em publicação. Os países desenvolvidos como EUA e os principais países europeus mantém seus investimentos em pesquisas científicas e tecnológicas, pois consideram que o apoio à ciência leva ao desenvolvimento tecnológico necessário para a manutenção da vida sustentável (Kuppermann, 1994). Nos resultados obtidos neste trabalho, os americanos são os que mais investem na tecnologia dos veículos subaquáticos, e consequentemente, o que os torna mais aptos e preparados a efetivar parcerias e a conseguir subsídios financeiros, 
governamentais ou privados, para suas pesquisas (Raskoff et al., 2010, Chadwick Jr et al., 2019). Países como Reino Unido e Alemanha também lideram como principais parceiros de pesquisa (Chen et al., 2020, Iacono et al., 2020).

Publicações envolvendo parcerias entre vários países representam menos de $50 \%$. O baixo número pode ser explicado pela dificuldade de colaboração entre instituições, falta ou limitação de financiamento e dificuldade de coordenação de pesquisas simultâneas em vários locais (Lima et al., 2019).

Diferente do que Kuppermann (1994) propõe ao dizer que a ciência é uma atividade financeiramente inviável para países em desenvolvimento, os resultados mostram o Brasil em constante avanço e notável investimento nos estudos com ROV. A presença de países em desenvolvimento no topo da lista, corrobora com Tollefson (2018) sobre a mudança de cenário na ciência e tecnologia dos países em desenvolvimento como Brasil e China que aumentaram em 89\% e 125\%, respectivamente, suas publicações na área da ciência e tecnologia nos últimos 10 anos.

O investimento nesta tecnologia pode vir através de órgãos governamentais (Chadwick Jr et al., 2019, Carminatto et al., 2020) ou privados (Vásquez et al., 2015, Aristizábal et al., 2016) sendo o ROV adquirido no mercado comercial internacional (Pereira-Filho et al., 2011, Carminatto et al., 2020) ou até mesmo sendo desenvolvido para fins específicos e dedicados (Wahab et al., 2020, Zhou \& Zhao, 2020). Tal investimento pode trazer benefícios não somente para as entidades e colaboradores diretamente envolvidos como pode favorecer a sociedade como um todo (NUTECMAR, 2020).

Como visto nos resultados deste trabalho, pouco mais de $60 \%$ dos trabalhos utilizando ROV são financiados. Isso pode ser explicado, por um lado, como um reconhecimento notório dos benefícios em investir nos ROVs e por outro lado, no fato de ser uma tecnologia mais custosa e com isso a necessidade de as pesquisas serem subsidiadas por financiamentos e parcerias.

Órgãos governamentais que financiam a pesquisa são impulsionados pela sociedade científica e possuem interesses que abrangem questões muito mais amplas do que os resultados obtidos em cada pesquisa financiada. A compensação pelo investimento, para órgãos governamentais, atinge benefícios não somente para a área de estudo do projeto como também para a sociedade como um todo. Além de possuírem mais recursos financeiros disponíveis e não necessitarem de resultados à curto prazo para retorno dos investimentos. Diferentemente do setor privado, que ao investir, está gerando expectativas de resultados para obter o retorno do investimento, seja em forma financeira, social ou ambiental.

As entidades privadas costumam investir em pesquisa e desenvolvimento de tecnologias em prol de uma aplicação específica que traga um retorno para os próprios processos. Por exemplo, uma usina nuclear investe em um veículo subaquático remoto para inspeções em reatores nucleares para aumentar a periodicidade de manutenção, aumentando a segurança no processo e reduzindo custos e tempo em suas operações. Já as organizações governamentais cumprem políticas públicas que exigem aportes constantes em ciência e tecnologia.

O aporte de recursos governamentais para ciência e tecnologia está relacionado ao PIB (Produto Interno Bruto) de cada país. A fração de investimento é muito maior para países mais desenvolvidos do que para países menos desenvolvidos (Kuppermann, 1994). A Fundação Nacional da Ciência (NSF) relata que, em 2019, os EUA investiram 2,7\% de seu PIB enquanto o Brasil investiu 1,3\% (Caires, 2020). Diante deste cenário, é importante avaliar que para os países em desenvolvimento, o esforço deve ser muito maior para que seja investido uma proporção maior de seu PIB em ciência e tecnologia para que estes alcancem maiores níveis de desenvolvimento (Kuppermann, 1994).

\subsection{Escolha dos ROVs, classificação e nacionalidade}

Fazendo a análise dos trabalhos estudados para a elaboração deste estudo, notamos que apenas 33\% dos pesquisadores optam por desenvolver ROVs próprios e, portanto, a maioria utiliza em suas pesquisas robôs subaquáticos já existentes no mercado. Essa escolha tende a ser motivada pela necessidade de busca por resultados compatíveis com os objetivos propostos 
de cada estudo e ambas as maneiras geram benefícios para seus países. Os pesquisadores que optam por utilizar ROVs comerciais (aqueles que são vendidos por empresas privadas) incentivam a indústria nacional ou beneficiam as relações de comércio exterior ao adquirirem equipamentos internacionais.

Os pesquisadores que optam por desenvolver suas tecnologias são motivados por demandas específicas e que, por questões variáveis, não justificam a aquisição de um equipamento já existente. Por exemplo, a estabilidade do ROV a precisão de seus movimentos desempenha um papel vital na eficácia de sua operação e, portanto, é relevante avaliar se a aplicação está sendo feita com o equipamento ideal e adequado para a execução do método proposto (Zarei et al., 2020). No Brasil, a burocracia de importação de produtos é rígida e, portanto, pode ser um dos motivos de ser o país que lidera nos estudos de desenvolvimento de ROVs próprios. Um ponto positivo deve ser destacado: com maior aporte de recursos financeiros, os pesquisadores brasileiros contribuem com o incentivo à pesquisa, à indústria e ao comércio que buscam acompanhar a evolução do mercado e são motivados a evoluir para manterem-se ativos em seus mercados.

Em segundo lugar de países que mais investem no desenvolvimento de seus próprios ROVs está a China com 15\%. Além de ser um país que vem apresentando números promissores de investimento em ciência, tecnologia e desenvolvimento, a China possui grande inteligência e mão de obra no desenvolvimento de produtos e faz uso dessas qualidades na produção de veículos subaquáticos e acessórios, especialmente voltados à indústria nuclear.

Entre os ROVs desenvolvidos por seus pesquisadores, a classe mais comum, acima de $80 \%$, é a classe de observação (OCROV) devido a seu baixo custo e alta aplicabilidade em ambientes diversos (Christ \& Wernli, 2014, NUTECMAR, 2020). É então na área de engenharia e tecnologia que encontramos $100 \%$ dos trabalhos que apresentam o desenvolvimento e construção dos ROVs próprios. Tal dado é concomitante à afirmação antes feita neste trabalho, de que o desenvolvimento de robôs subaquáticos próprios é motivado por uma demanda específica da indústria e trás benefícios, não só a seus usuários diretos como à movimentação socioeconômica do país, através do incentivo à estudo, pesquisa, desenvolvimento, tecnologias e comércio.

Os grandes produtores comerciais de ROV estão distribuídos no continente Europeu e nos EUA. Por esse motivo, países como França, Itália, Reino Unido e Estados Unidos aparecem no topo da lista com trabalhos que utilizam ROVs existentes. A tecnologia comercializada já é ativa e muito bem-produzida pela indústria local e, portanto, esses pesquisadores não veem a necessidade de explorar os conhecimentos na área de desenvolvimento, mas sim, nos pioneirismos de resultados de pesquisas científicas. E por essa razão, $85,5 \%$ das pesquisas realizadas com ROVs que já existem no mercado são da área de ciências ambientais. $\mathrm{O}$ investimento à ciência e à tecnologia é focada na exploração dos recursos naturais e nas pesquisas científicas ambientais.

\section{Conclusão}

Com base na análise apresentada ao longo da discussão deste trabalho, pode-se concluir que o avanço do conhecimento em veículos subaquáticos operados remotamente foi alavancado pelo crescente número de publicações científicas. Independentemente de os projetos publicados terem sido financiados, com subsídios próprios ou em parcerias, a inserção e constante permanência da tecnologia no meio científico trouxe inúmeros benefícios para a sociedade. Seja no âmbito socioeconômico por meio do incentivo à indústria e ao conhecimento tecnológico específico, seja no quesito ambiental por meio da difusão de técnicas não invasivas e cada vez mais sustentáveis e seguras que consolidam a busca pelo conhecimento e pelo inexplorável.

Os investimentos provenientes de órgãos governamentais trazem aos países que o fazem benefícios ligados a políticas públicas e à sociedade. Investir em pesquisa é investir em ciência, em conhecimento e na difusão da educação científica que leva os países a maiores patamares de desenvolvimento e consequentemente conseguem investir mais, criando um ciclo de 
crescimento e investimento. As pesquisas ambientais são os principais focos de investimento de entidades governamentais pois trazem respostas à comunidade científica que consegue estender para a sociedade os benefícios do desenvolvimento científico.

No que tange aos investimentos privados, o panorama trouxe um incentivo à indústria, pois apontou ser viável a inserção de recursos financeiros para pesquisa e desenvolvimento de ferramentas que atendam às demandas do mercado atual. Além de obterem benefícios para seus processos, geram empregos, contribuem para a economia nacional e ainda incentivam à pesquisa, o conhecimento e a ciência.

Atualmente, existem veículos subaquáticos denominados, pelo mercado, de drones subaquáticos que apresentam tamanho reduzido e custo consideravelmente mais baixo do que um veículo profissional. Dependendo do objetivo do estudo, este recurso pode ser considerado quando a pesquisa possui limitações financeiras ou escassez de veículos subaquáticos profissionais. A possibilidade de locação e aquisição desses veículos é plausível para a viabilidade de projetos científicos que utilizem essas tecnologias como metodologia.

\section{Agradecimentos}

Agradecimento a Coordenação de Aperfeiçoamento de Pessoal de Nível Superior - Brasil (CAPES) - Código de Financiamento 001 pela bolsa concebida a primeira autora. Agradecimentos especiais ao Patrick Martins, que engrandeceu o trabalho com a ilustração dos mapas e à Paula Martins Escudeiro pela revisão e considerações com nosso trabalho. Agradecemos também ao Núcleo de Tecnologia Marinha e Ambiental (NUTECMAR) pelas informações e conhecimento passado.

\section{Referências}

Alcoforado, V., Marques, P. C., \& Silva-Cavalcanti, J. S. (2013). Protótipo de ROV (Remotely Operated Vehicles) e suas aplicações em águas do semiárido: resultados preliminares. [apresentação em conferência]. XIII Jornada De Ensino, Pesquisa e Extensão, JEPEX 2013, UFRPE: http://www.eventosufrpe.com.br/2013/cd/resumos/R1185-1.pdf

Aristizábal, L. M., Rúa, S., Gaviria, C. E., Osorio, S. P., Zuluaga, C. A., Posada, N. L., \& Vásquez, R. E. (2016). Design of an open source-based control platform for an underwater remotely operated vehicle. Dyna, 83(195), 198-205. https://doi.org/10.15446/dyna.v83n195.49828

Boutteau, R., Rossi, R., Qin, L., Merriaux, P., \& Savatier, X. (2020). A vision-based system for robot localization in large industrial environments. Journal of Intelligent \& Robotic Systems, 99(2), 359-370. https://doi.org/10.1007/s10846-019-01114-X

Caires, L. (2020). Nos países desenvolvidos, o dinheiro que financia a ciência na universidade é público. Jornal da USP. Disponível em:https://jornal.usp.br/ciencias/nos-paises-desenvolvidos-o dinheiro-que-financia-a-ciencia-e-publico/. Acessado em: 10 de set. 2020.

Cánovas-Molina, A., Montefalcone, M., Bavestrello, G., Cau, A., Bianchi, C.N., Morri, C., Canese, S., \& Bo, M. (2016). A new ecological index for the status of mesophotic megabenthic assemblages in the Mediterranean based on ROV photography and video footage. Continental Shelf Research, 121, 13-20. https://doi.org/10.1016/J.CSR.2016.01.008

Carminatto, A. A. (2019). Complexidade do hábitat, caracterização e diversidade de peixes recifais da Ilha das Palmas e da Ilha do Mato (Guarujá/SP). [Dissertação de mestrado, Universidade Santa Cecília, Santos, Brasil]. https://unisanta.br/arquivos/mestrado/ecologia/dissertacoes/D issertacao_AMANDAAPARECIDACARMINATTO333.pdf

Carminatto, A.A., Rotundo, M.M., Butturi-Gomes, D., Barrella, W., \& Junior, M. P. (2020). Effects of habitat complexity and temporal variation in rocky reef fish communities in the Santos estuary (SP), Brazil. Ecological Indicators, 108, 105728. https://doi.org/10.1016/j.ecolind.2019.105728

Centeno, M. L. (2007). Rovfurg-ii: Projeto e construção de um veículo subaquático não tripulado de baixo custo. [Dissertação de Mestrado, Universidade Federal do Rio Grande, Rio Grande, Brasil]. http://repositorio.furg.br/bitstream/handle/1/3480/Projeto\%20e \%20constru\%C3\%A7\%C3\%A3o\%20de\%20um\%20ve\%C3\%ADculo\%20subaqu\%C3\%A1 tico\%20n\%C3\%A3o\%20tripulado\%20de\%20baixo\%20custo.pdf? sequence $=1$

Chadwick Jr, W. W., Rubin, K. H., Merle, S. G., Bobbitt, A. M., Kwasnitschka, T., \& Embley, R. W. (2019). Recent eruptions between 2012-2018 discovered at West Mata submarine volcano (NE Lau Basin, SW Pacific) and characterized by new ship, AUV, and ROV data. Frontiers in Marine Science, 6, 495. https://doi.org/10.3389/fmars.2019.00495

Chen, T. T., Paull, C. K., Liu, C. S., Klaucke, I., Hsu, H. H., Su, C. C., Gwiazda, R., \& Caress, D. W. (2020). Discovery of numerous pingos and cometshaped depressions offshore southwestern Taiwan. Geo-Marine Letters, 1-15. https://doi.org/10.1007/s00367-019-00577-z

Cho, B. H., Byun, S. H., Shin, C. H., Yang, J. B., Song, S. I., \& Oh, J. M. (2004). KeproVt: underwater robotic system for visual inspection of nuclear reactor internals. Nuclear Engineering and Design, 231(3), 327-335. https://doi.org/10.1016/j.nucengdes.2004.03.012 
Christ, R. D., \& Wernli SR, R. L. (2014). The ROV manual: a user guide for remotely operated vehicles (2ºd.). Oxforf: Butterworth-Heinemann, 2014.

Cooke, N. J. (2006). Human factors of remotely operated vehicles. Proceedings of the Human Factors and Ergonomics Society Annual Meeting, 50(1), 166169. https://doi.org/10.1177/154193120605000135

Gao, H., Jiao, X., Zhou, C., Shen, Q., \& Yu, Y. (2011). Study on remote control underwater welding technology applied in nuclear power station. Procedia Engineering, 15, 4988-4993. https://doi.org/10.1016/j.proeng.2011.08.927

Hartill, É. C., Waller, R. G., \& Auster, P. J. (2020). Deep coral habitats of Glacier Bay National Park and Preserve, Alaska. PloS one, 15 (8), e0236945. https://doi.org/10.1371/journal.pone.0236945

Ho, G., Pavlovic, N., \& Arrabito, R. (2011). Human factors issues with operating unmanned underwater vehicles. Human Factors and Ergonomics Society Annual Meeting Proceedings, 55(1). https://doi.org/10.1177/1071181311551088

Htun, T. Z., Suzuki, H., \& García-Vallejo, D. (2020). Dynamic modeling of a radially multilayered tether cable for a remotely-operated underwater vehicle (ROV) based on the absolute nodal coordinate formulation (ANCF). Mechanism and Machine Theory, 153, 103961. https://10.1016/j.mechmachtheory.2020.103961

Hughes, S. J. M., Jones, D. O. B., Hauton, C., Gates, A. R., \& Hawkins, L. E. (2010). An assessment of drilling disturbance on Echinus acutus var. norvegicus based on in-situ observations and experiments using a remotely operated vehicle (ROV). Journal of Experimental Marine Biology and Ecology, 395(1-2), 3747, 2010. https://10.1016/J.JEMBE.2010.08.012

Iacono, C. L., Guillén, J., Guerrero, Q., Durán, R., Wardell, C., Hall, R. A., Aslam, T., Carterd,G. D. O., Galese, J., \& Huvenne, V. A. (2020). Bidirectional bedform fields at the head of a submarine canyon (NE Atlantic). Earth and Planetary Science Letters, 542, 116321. https://doi.org/10.1016/j.epsl.2020.116321

Johansen, C., Macelloni, L., Natter, M., Silva, M., Woosley, M., Woolsey, A.,Diercks, A.R., Hill, J., Viso, R., Marty, E., Lobodin, V.V., Shedd, W., Joye, S.B., I.R. MacDonald, I.R., \& Lobodin, V.V. (2020). Hydrocarbon migration pathway and methane budget for a Gulf of Mexico natural seep site: Green Canyon 600. Earth and Planetary Science Letters, 545, 116411. https://doi.org/10.1016/j.eps1.2020.116411

Koji, K. (1999). Underwater inspection robot-AIRIS 21®. Nuclear Engineering and Design, 188(3), 367-371. https://doi.org/10.1016/S0029-5493(99)00045$\mathrm{X}$

Kuppermann, A. (1994). Investimentos em ciência e tecnologia. Estudos Avançados, 8(20), 18-22. https://doi.org/10.1590/S0103-40141994000100005

Lapa, J. M., Pereira, F. D. S., de Cerqueira, E. V. G., de de Santana, D. E., \& de Jesus, C. A. (2012). Aprimoramento na construção de um minissubmarino de monitoramento não tripulado de baixo custo. [apresentação em conferência]. VII CONNEPI-Congresso Norte Nordeste de Pesquisa e Inovação. https://propi.ifto.edu.br/ocs/index.php/connepi/vii/paper/viewFile/1746/2484

Lee, S. U., Choi, Y. S., Jeong, K. M., \& Jung, S. (2006). Development of a Tele-operated Underwater Robotic System for maintaining a light-water type power reactor. 2006 IEEE SICE-ICASE International Joint Conference, 3017-3021. https://doi.org/10.1109/SICE.2006.315148

Lemaire, I. P. (1988). NOSC and Remotely Operated Vehicles (ROVs) and Autonomous Unmanned Vehicles (AUVs). Naval Ocean Systems Center: San Diego, CA, USA. https://apps.dtic.mil/sti/citations/ADA203356

Lima, J. S., Zalmon, I. R., \& Love, M. (2019). Overview and trends of ecological and socioeconomic research on artificial reefs. Marine Environmental Research, 145, 81-96. https://doi.org/10.1016/j.marenvres.2019.01.010

Linley, T. D., Alt, C. H., Jones, D. O., \& Priede, I. G. (2013). Bathyal demersal fishes of the Charlie-Gibbs Fracture Zone region (49 ${ }^{\circ}-54^{\circ}$ N) of the MidAtlantic Ridge: III. Results from remotely operated vehicle (ROV) video transects. Deep Sea Research Part II: Topical Studies in Oceanography, 98, 407-411. https://doi.org/10.1016/j.dsr2.2013.08.013

Lorance, P., \& Trenkel, V. M. (2006). Variability in natural behaviour, and observed reactions to an ROV, by mid-slope fish species. Journal of Experimental Marine Biology and Ecology, 332(1), 106-119. https://doi.org/10.1016/j.jembe.2005.11.007

Luo, Y., Tao, J., Sun, Q., Deng, L., \& Deng, Z. (2018). A new underwater robot for crack welding in nuclear power plants. 2018 IEEE International Conference on Robotics and Biomimetics (ROBIO), 77-82. https://doi.org/10.1109/ROBIO.2018.8665279

Lv, X. M., Liu, Y. F., Gao, H. B., Ding, L., Tao, J. G., Xia, K. R., \& Deng, Z. Q. (2014). Design of underwater welding robot used in nuclear plant. Key Engineering Materials, 620, 484-489. https://doi.org/10.4028/www.scientific.net/KEM.620.484

Manoukian, S., Fabi, G., \& Naar, D.F. (2011). Multibeam investigation of an artificial reef settlement in the adriatic sea (Italy) 33 years after its deployment. Brazilian Journal of Oceanography, 59(SPE1), 145-153. https://doi.org/10.1590/S1679-87592011000300016

NUTECMAR - Núcleo de Tecnologia Marinha e Ambiental, (2020). ROV Training - Nível 1 (1ºd.). Apostila didática: São Paulo.

Park, J.Y., Cho, B. H., \& Lee, J.K. (2009). Trajectory-tracking control of underwater inspection robot for nuclear reactor internals using Time Delay Control. Nuclear Engineering and Design, 239(11), 2543-2550. https://doi.org/10.1016/j.nucengdes.2009.07.029

Pereira-Filho, G. H., Amado-Filho, G. M., Guimarães, S. M., Moura, R.L., Sumida, P. Y., Abrantes, D. P., Bahia, R. G., Güth, A. Z., Jorge, R. R., \& Francini Filho, R. B. (2011). Reef fish and benthic assemblages of the Trindade and Martin Vaz island group, southwestern Atlantic. Brazilian Journal of Oceanography, 59(3), 201-212. https://doi.org/10.1590/S1679-87592011000300001

Raskoff, K. A., Hopcroft, R. R., Kosobokova, K. N., Purcell, J. E., \& Youngbluth, M. (2010). Jellies under ice: ROV observations from the Arctic 2005 hidden ocean expedition. Deep Sea Research Part II: Topical Studies in Oceanography, 57(1-2), 111-126. https://doi.org/10.1016/J.DSR2.2009.08.010 
Research, Society and Development, v. 10, n. 12, e396101220462, 2021

(CC BY 4.0) | ISSN 2525-3409 | DOI: http://dx.doi.org/10.33448/rsd-v10i12.20462

Souza, M. T. de, Silva, M. D. da, \& Carvalho, R. de. (2010). Integrative review: what is it? How to do it? Einstein (São Paulo), 8 (1), $102-106$. https://doi.org/10.1590/s1679-45082010rw1134

Souza, W. L. de (2010). Sistema de propulsores para um ROV-subaquático. [Trabalho de Conclusão de Curso, Centro Universitário das Faculdades Associadas de Ensino, São João da Boa Vista, Brasil]. https://docplayer.com.br/7897932-Centro-universitario-das-faculdades-associadas-de-ensino-sistemade-propulsores-para-um-rov-subaquatico.html

Smolowitz, R. J., Patel, S. H., Haas, H. L., \& Miller, S. A. (2015). Using a remotely operated vehicle (ROV) to observe loggerhead sea turtle (Caretta caretta) behavior on foraging grounds off the mid-Atlantic United States. Journal of Experimental Marine Biology and Ecology, 471, 84-91. https://doi.org/10.1016/j.jembe.2015.05.016

Tahir, A. M., \& Iqbal, J. (2014). Underwater robotic vehicles: latest development trends and potential challenges. Science International, 26 (3), 2014. http://www.sci-int.com/pdf/636639120492572343.pdf

Tehrani, N. H., Heidari, M., Zakeri, Y., \& Ghaisari, J. (2010). Development, depth control and stability analysis of an underwater Remotely Operated Vehicle (ROV). IEEE ICCA. https://doi.org/10.1109/ICCA.2010.5524051

Tollefson, J. (2018). China declared largest source of research articles. Nature, 553(7689), 390-39. https://doi.org/10.1038/d41586-018-00927-4

Vásquez, R. E., Correa, J. C., Ramírez-Macías, J. A., Taborda, E. A., Zuluaga, C. A., Posada, N. L., \& Londoño, J. M. (2015). Una arquitectura para el diseño conceptual de vehículos para exploración subacuática. Ingeniería y Ciencia, 11(21), 73-97. https://doi.org/10.17230/ingciencia.11.21.4

Wahab, I. H. A., Nuryaningsih, R. E., \& Sardju, A. P. (2020). Proposed Mathematical Modeling of Small Remotely Operated Vehicle (ROV) Movement. Journal of Physics: Conference Series, 1569, (4), 042002. https://doi.org/10.1088/1742-6596/1569/4/042002

Zarei, A., Ashouri, A., Hashemi, S. M. J., Bushehri, S. F., Izadpanah, E., \& Amini, Y. (2020). Experimental and numerical study of hydrodynamic performance of remotely operated vehicle. Ocean Engineering, 212, 107612. https://doi.org/10.1016/j.oceaneng.2020.107612

Zhang, X., Zhang, J., Yuan, J. \& Li, M. (2013). Development of an underwater robot for nuclear reactor vessel. 2013 IEEE International Conference on Robotics and Biomimetics (ROBIO), 1699-1703. https://doi.org/10.1109/ROBIO.2013.6739712

Zhou, B., \& Zhao, M. (2020). Numerical simulation of thruster-thruster interaction for ROV with vector layout propulsion system. Ocean Engineering, 210, 107542. https://doi.org/10.1016/j.oceaneng.2020.107542 